\title{
OPTICAL SPECTROSCOPY OF WR STARS IN M33 AND M31
}

\author{
H. SCHILD, L.J. SMITH \& A.J. WILLIS \\ Department of Physics and Astronomy \\ University College London \\ Gower Street, London WC1E 6BT \\ England
}

\begin{abstract}
We present new optical spectroscopy obtained with the Faint Object Spectrograph on the William Herschel Telescope of 6 WR stars in the local group galaxy M33 and $6 \mathrm{WR}$ stars in M31. These spectra cover the wavelength range $\lambda \lambda 3500-9750 \AA$. We confirm the previous WR classifications of the M31 stars. In the M33 sample, 4 stars are classified as WC4-5, one is confirmed as WN-WCE and a further WN-WCE star has been identified. The widths of the CIV $\lambda 5800$ emission differ by a factor of three amongst the M33 WC4-5 sample, spanning values exhibited by galactic WC8-WC4 stars and confirming the existence of narrow-lined WC4-5 stars in M33. We find that the M33 WCE line widths appear to be correlated with galactocentric distance and loosely connected to ambient metallicity. The narrowest lined WCE star MC 53 is in an unusually high (above solar) metallicity region. Further observations of a larger sample of M33 stars are required to confirm these preliminary results.
\end{abstract}

The full version of this paper is to be published in Astronomy and Astrophysics. 